\title{
A Synthesis of the Welwistatin Core (supporting information)
}

\author{
Ryan Lauchli and Kenneth J. Shea* \\ Department of Chemistry, 1102 Natural Sciences II, University of California, Irvine, \\ Irvine, CA 92697-2025
}

Instrumentation: NMR measurements were conducted with Bruker instruments at 500 $\mathrm{MHz}$ for proton spectra and $125 \mathrm{MHz}$ for carbon spectra. Chemical shifts are given in ppm values referenced to residual solvent peaks.

General Procedures: Toluene, THF, and dichloromethane were purified by passage through alumina columns. Isopropanol, triethylamine, and benzene were distilled from $\mathrm{CaH}_{2}$. Methanol was distilled from its magnesium alkoxide. DMF was dried over $4 \AA$ molecular sieves. All reactions were performed under $\mathrm{N}_{2}$ using flame dried glassware and standard syringe and septa techniques. Removal of solvent in vacuo refers to use of a Büchi rotary evaporator. Reagents were purchased from VWR, Fisher, or Aldrich and generally used without purification. TLC was performed with aluminum-backed silica gel $60 \mathrm{~F}_{254}$ analytical plates from EMD. Visualization of compound spots was done with a short wave UV lamp followed by staining with either anisaldehyde or Seebach's stain (phosphomolybdic acid and $\left.\mathrm{Ce}_{2}\left(\mathrm{SO}_{4}\right)_{3}\right)$. Flash chromatography was performed on Geduran silica gel $60(40-63 \mu \mathrm{m})$ from EMD.

\section{4-bromo-1H-indole-3-carbaldehyde (6)}

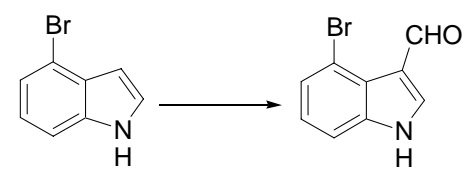

A round bottom flask was charged with $6 \mathrm{~mL}$ of dry DMF, and the flask was cooled in an ice bath. The liquid was magnetically stirred under $\mathrm{N}_{2}$ and $\mathrm{POCl}_{3}(0.98 \mathrm{~mL}, 10.5 \mathrm{mmol})$ was added dropwise. The solution was stirred for $2 \mathrm{~min}$, and then a solution of 4- 
bromoindole $^{1}$ (0.94 g, $\left.4.7 \mathrm{mmol}\right)$ in $5 \mathrm{~mL}$ of DMF was added dropwise. The ice bath was removed and the reaction mixture was left to stir for $1 \mathrm{~h}$ at ambient temp. The reaction mixture became a heavy suspension, and was slowly quenched with $2.66 \mathrm{~g} \mathrm{KOH}$ in 10 $\mathrm{mL}$ of water. The quench was done slowly enough to keep the reaction mixture just below boiling. The reaction mixture was left to cool overnight, and was then partitioned between EtOAc and saturated aqueous $\mathrm{NaHCO}_{3}$. The organic layer was washed with saturated aqueous $\mathrm{NaCl}$, dried over $\mathrm{Na}_{2} \mathrm{SO}_{4}$, and concentrated in vacuo to give an offwhite solid. Yield: 1.11 g, quantitative. NMR spectral data matched literature values. ${ }^{2}$

\section{4-Trimethylsilanylethynyl-1H-indole-3-carbaldehyde (7)}

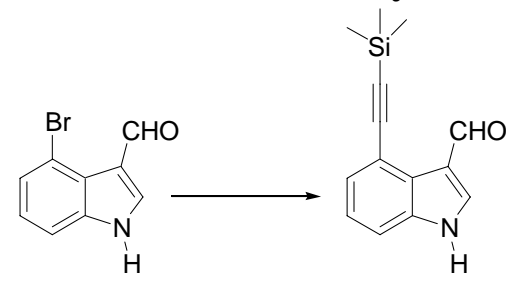

4-bromo-1H-indole-3-carbaldehyde (305 mg, $1.36 \mathrm{mmol}), \mathrm{Pd}\left(\mathrm{PPh}_{3}\right)_{4}(62.5 \mathrm{mg}, 0.05$ $\mathrm{mmol}$ ), and CuI (19 mg, $0.10 \mathrm{mmol}, 99.999 \%$ purity) were combined in a resealable glass flask in an Ar atmosphere using a glovebox. The flask was taken out of the glovebox, and triethylamine $(6.5 \mathrm{~mL})$ and trimethylsilylacetylene $(0.30 \mathrm{~mL}, 0.20 \mathrm{mmol})$ were added via syringe. A magnetic stir bar was added and the reaction was sealed. The reaction was heated to $100{ }^{\circ} \mathrm{C}$ with an oil bath and stirring was maintained. After $22 \mathrm{~h}$, the now dark reaction was allowed to cool and then diluted with EtOAc $(100 \mathrm{~mL})$. The mixture was filtered through celite 545. The organic solution was washed with saturated aqueous $\mathrm{NH}_{4} \mathrm{Cl}(3 \times 30 \mathrm{~mL})$, water $(3 \times 30 \mathrm{~mL})$, and brine $(30 \mathrm{~mL})$. The organics were dried over $\mathrm{Na}_{2} \mathrm{SO}_{4}$, filtered, adsorbed on celite 545, and the solvents were removed in vacuo. The impregnated celite was placed on top of a $\mathrm{SiO}_{2}$ flash chromatography column and eluted with 2:1 hexanes:EtOAc. The product was collected as a tan solid. Yield: $321 \mathrm{mg}, 98 \%$. Mp: $148.8-149.8^{\circ} \mathrm{C}$

${ }^{1} \mathrm{H}$ NMR (d6-DMSO, 500 MHz) 12.48 (s.br., 1 H), 10.75 (s, 1 H), 8.26 (s, 1 H), 7.59 (dd, $J=8.2,0.8 \mathrm{~Hz}, 1 \mathrm{H}$ ), 7.37 (dd, $J=6.8,0.98 \mathrm{~Hz}, 1 \mathrm{H}$ ), 7.23 (app.t, $J=7.8 \mathrm{~Hz}, 1 \mathrm{H}$ ), 0.27 (s, $9 \mathrm{H}) .{ }^{13} \mathrm{C}$ NMR (d6-DMSO, $\left.125 \mathrm{MHz}\right)$ 185.0, 136.7, 132.4, 126.8, 125.7, 122.5, 118.0, 114.3, 112.5, 105.5, 97.8, -0.28. IR (KBr, cm $\left.{ }^{-1}\right)$ : 3267, 3117, 3050, 2959, 2895, 2848, 2157, 2144, 1652, 1636, 1606, 1514, 1504, 1446, 1385, 1338, 1322, 1300, 1250 , 1121, 865, 844, 785, 760, 731, 652. HRMS (TOF ES+) calculated for $\mathrm{C}_{14} \mathrm{H}_{15} \mathrm{OSiNNa}$ $(\mathrm{M}+\mathrm{Na})^{+}$264.0821, observed 264.0825.

\footnotetext{
${ }^{1}$ Available commercially, but made in this case according to: Moyer, M. P.; Shiurba, J. F.; Rapoport, H. J. Org. Chem. 1986, 51, 5106.

2 Somei, M.; Kizu, K.; Kunimoto, M.; Yamada, F. Chem. Pharm. Bull. 1985, 33, 3696.
} 


\section{4-Ethynyl-1H-indole-3-carbaldehyde}

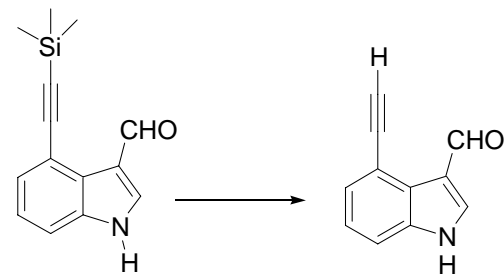

A mixture of 4-trimethylsilanylethynyl- $1 \mathrm{H}$-indole-3-carbaldehyde (321 mg, $1.33 \mathrm{mmol}$ ) and $\mathrm{K}_{2} \mathrm{CO}_{3}\left(25 \mathrm{mg}\right.$ ) was stirred in $6 \mathrm{~mL}$ of methanol under $\mathrm{N}_{2}$ for $18 \mathrm{~h}$. The solvent was then removed in vacuo, and the residue was partitioned between EtOAc (45 mL) and saturated aqueous $\mathrm{NaHCO}_{3}$. The organic layer was washed with water and brine, dired over $\mathrm{Na}_{2} \mathrm{SO}_{4}$, filtered, and concentrated in vacuo to give the product as a tan solid. Yield: $193 \mathrm{mg}, 86 \%$.

Mp: $158.0-158.9^{\circ} \mathrm{C}$.

${ }^{1}$ H NMR (d6-DMSO, 500 MHz) 12.49 (s.br., 1 H), 10.73 (s, 1 H), 8.27 (s, 1 H), 7.60 (dd, $J=8.3,0.73 \mathrm{~Hz}, 1 \mathrm{H}$ ), 7.40 (dd, $J=7.3,0.73 \mathrm{~Hz}, 1 \mathrm{H}$ ), 7.24 (app.t, $J=7.8 \mathrm{~Hz}, 1 \mathrm{H}$ ), 4.50 (s, $1 \mathrm{H}) .{ }^{13} \mathrm{C}$ NMR (d6-DMSO, $\left.125 \mathrm{MHz}\right)$ 185.0, 136.8, 132.5, 127.2, 125.8, 122.6, 117.9, 114.2, 112.1, 84.2, 83.7. IR (KBr, cm$\left.{ }^{-1}\right)$ : 3286, 3272, 3231, 3117, 3091, 3049, 3006, 2944, 2890, 2869, 1656, 1638, 1519, 1503, 1389, 1335, 1300, 1131, 788, 737. HRMS (TOF ES+) calculated for $\mathrm{C}_{11} \mathrm{H}_{7} \mathrm{ONNa}(\mathrm{M}+\mathrm{Na})^{+}$192.0425, observed 192.0419.

\section{4-Ethynyl-1-(toluene-4-sulfonyl)-1H-indole-3-carbaldehyde (8)}

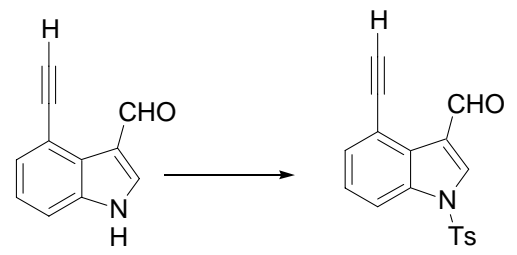

In a round bottom flask under $\mathrm{N}_{2}$, 4-ethynyl- $1 \mathrm{H}$-indole-3-carbaldehyde (100 mg, 0.59 mmol), pTSCl (133 mg, $0.70 \mathrm{mmol}$ ), DMAP (5 mg), and triethylamine (123 $\mu \mathrm{L}, 0.92$ mmol) were dissolved in $5 \mathrm{~mL}$ of $\mathrm{CH}_{2} \mathrm{Cl}_{2}$, and the solution was stirred for $2.5 \mathrm{~h}$. The reaction mixture was diluted with more $\mathrm{CH}_{2} \mathrm{Cl}_{2}$, and the organics were washed with $1 \mathrm{~N}$ $\mathrm{HCl}$. The organic layer was dried over $\mathrm{Na}_{2} \mathrm{SO}_{4}$, filtered, adsorbed onto celite 545, and the solvents were removed in vacuo. The product was isolated by $\mathrm{SiO}_{2}$ flash chromatography using 3:1 hexanes:EtOAc to give the product as an off-white solid. Yield: $163 \mathrm{mg}, 85 \%$. Mp: $162.0-163.1^{\circ} \mathrm{C}$.

${ }^{1} \mathrm{H} \mathrm{NMR}\left(\mathrm{CDCl}_{3}, 500 \mathrm{MHz}\right) 10.90$ (s, $\left.1 \mathrm{H}\right), 8.39$ (s, $\left.1 \mathrm{H}\right), 8.04$ (d, $\left.J=8.3 \mathrm{~Hz}, 1 \mathrm{H}\right), 7.83$ $(\mathrm{d}, J=8.6 \mathrm{~Hz}, 2 \mathrm{H}), 7.52$ (d, $J=7.6 \mathrm{~Hz}, 1 \mathrm{H}), 7.34$ (t, $J=8.0 \mathrm{~Hz}, 1 \mathrm{H}), 7.29$ (d, $J=8.1$ Hz, $2 \mathrm{H}), 3.43$ (s, $1 \mathrm{H}), 2.37$ (s, $3 \mathrm{H}) .{ }^{13} \mathrm{C} \mathrm{NMR}\left(\mathrm{CDCl}_{3}, 125 \mathrm{MHz}\right)$ 186.9, 146.5, 135.3, 134.4, 131.4, 130.5, 130.2, 128.2, 127.5, 125.3, 122.2, 114.8, 114.4, 82.7, 21.8. IR (KBr, $\mathrm{cm}^{-1}$ ): 3278, 3136, 2871, 1676, 1594, 1531, 1485, 1423, 1405, 1381, 1372, 1289, 1273, 
1189, 1181, 1174, 1146, 1096, 1085, 1061, 1043, 878, 821, 787, 745, 704, 689, 666, 639, 600, 577, 549. HRMS (TOF ES+) calculated for $\mathrm{C}_{18} \mathrm{H}_{14} \mathrm{O}_{3} \mathrm{NS}(\mathrm{M}+\mathrm{H})^{+} 324.0694$, observed 324.0699.

\section{4-(1-Methylene-allyl)-1-(toluene-4-sulfonyl)-1H-indole-3-carbaldehyde (9)}

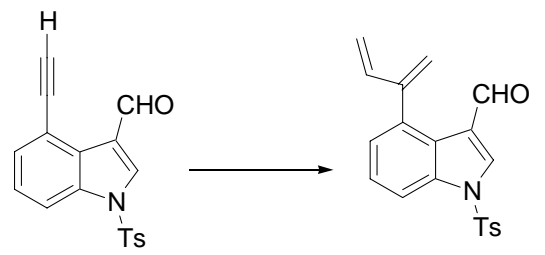

A 2-dram vial with a magnetic stir bar was introduced into the glovebox under $\mathrm{N}_{2}$, and was charged with 4-ethynyl-1-(toluene-4-sulfonyl)-1H-indole-3-carbaldehyde (49 mg, $0.15 \mathrm{mmol}$ )and Grubb’s $2^{\text {nd }}$ generation catalyst $(6 \mathrm{mg}, 0.0076 \mathrm{mmol})$. The septum-sealed vial was taken out of the glovebox and an ethylene balloon was used to provide positive ethylene pressure. Toluene $(2.5 \mathrm{~mL})$ was added, and an outlet needle was used to briefly purge the atmosphere with ethylene. The solution was heated to $75-80{ }^{\circ} \mathrm{C}$ for $40 \mathrm{~min}$, in which time the solution became slightly darker. The reaction was quenched with 5 drops of ethylvinyl ether, and the solvents were removed in vacuo. The residue was purified by $\mathrm{SiO}_{2}$ flash chromatography using 3:1 hexanes:EtOAc to give the diene as a brown oil. Yield: $50 \mathrm{mg}$, 94\%.

${ }^{1} \mathrm{H} \mathrm{NMR}\left(\mathrm{CDCl}_{3}, 500 \mathrm{MHz}\right) 10.10$ (s, $\left.1 \mathrm{H}\right), 8.32$ (s, $\left.1 \mathrm{H}\right), 7.98$ (dd, $J=8.5,0.98 \mathrm{~Hz}, 1$ H), 7.88 (d, $J=8.3 \mathrm{~Hz}, 2 \mathrm{H}), 7.38$ (dd, $J=8.3,7.3 \mathrm{~Hz}, 1 \mathrm{H}), 7.32$ (d, $J=8.3 \mathrm{~Hz}, 2 \mathrm{H}$ ), 7.15 (dd, $J=7.3,0.98 \mathrm{~Hz}, 1 \mathrm{H}$ ), 6.67 (dd, $J=17.4,10.5 \mathrm{~Hz}, 1 \mathrm{H}$ ), 5.49 (d, $J=0.98 \mathrm{~Hz}, 1$ H), 5.28 (s, $1 \mathrm{H}), 5.16$ (d, $J=10.3 \mathrm{~Hz}, 1 \mathrm{H}), 4.68$ (d, $J=17.4 \mathrm{~Hz}, 1 \mathrm{H}), 2.39(\mathrm{~s}, 3 \mathrm{H}) .{ }^{13} \mathrm{C}$ NMR $\left(\mathrm{CDCl}_{3}, 125 \mathrm{MHz}\right)$ 187.3, 148.0, 146.3, 138.4, 135.2, 134.6, 133.4, 131.3, 130.5, 127.6, 125.99, 125.89, 125.59, 121.9, 119.9, 118.7, 113.0, 21.9. IR ( $\left.\mathrm{NaCl}, \mathrm{cm}^{-1}\right)$ : 3134, 3090, 3053, 3036, 3006, 2924, 2872, 1682, 1596, 1531, 1488, 1406, 1378, 1286, 1274, 1190, 1177, 1161, 1139, 1096, 1088, 1041, 1026, 991, 912, 815, 798, 755, 738, 703, 687, 665, 629. HRMS (TOF ES+) calculated for $\mathrm{C}_{20} \mathrm{H}_{17} \mathrm{O}_{3} \mathrm{NSNa}(\mathrm{M}+\mathrm{Na})^{+} 374.0827$, observed 374.0828.

1-[4-(1-Methylene-allyl)-1-(toluene-4-sulfonyl)-1H-indol-3-yl]-prop-2-en-1-ol (10)

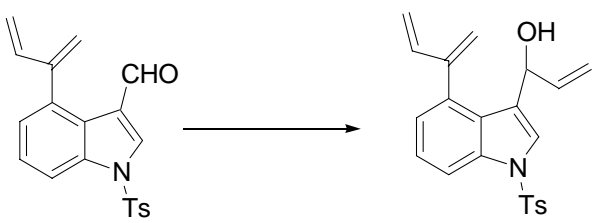


The aldehyde (96 mg, $0.27 \mathrm{mmol}$ ) was dissolved in $0.5 \mathrm{~mL}$ of THF under $\mathrm{N}_{2}$ in a 2-dram vial, and was magnetically stirred. The solution was cooled in an ice bath as vinylmagnesium bromide $(0.35 \mathrm{~mL}$ of a $1 \mathrm{M}$ solution in THF, $0.35 \mathrm{mmol})$ was added dropwise. The reaction mixture was stirred at ambient temp for $10 \mathrm{~min}$, and then was quenched with saturated aqueous $\mathrm{NH}_{4} \mathrm{Cl}$. The product was extracted into EtOAc, washed with brine, and dried over $\mathrm{Na}_{2} \mathrm{SO}_{4}$. The organics were concentrated in vacuo, and the residue was purified by $\mathrm{SiO}_{2}$ flash chromatography using 3:1 hexanes:EtOAc to give the product as a yellow tinted tar. Yield: $84 \mathrm{mg}, 81 \%$.

${ }^{1} \mathrm{H} \mathrm{NMR}\left(\mathrm{CDCl}_{3}, 500 \mathrm{MHz}\right) 7.96$ (dd, $\left.J=8.4,1.0 \mathrm{~Hz}, 1 \mathrm{H}\right), 7.80(\mathrm{~d}, J=8.5 \mathrm{~Hz}, 2 \mathrm{H})$, 7.57 (s, $1 \mathrm{H}$ ), 7.30 (dd, $J=8.3,7.3 \mathrm{~Hz}, 1 \mathrm{H}$ ), 7.26 (d, $J=8.3 \mathrm{~Hz}, 2 \mathrm{H}$ ), 7.00 (dd, $J=7.3$, $0.98 \mathrm{~Hz}, 1 \mathrm{H}$ ), 6.75 (dd, $J=17.4,10.5 \mathrm{~Hz}, 1 \mathrm{H}$ ), 6.12 (ddd, $J=15.4,10.5,4.9 \mathrm{~Hz}, 1 \mathrm{H}$ ), 5.52 (d, $J=0.98$ Hz, 2 H), 5.50 (s, 1 H), 5.40 (d, $J=17.4$ Hz, 1 H), 5.18-5.27 (m, $3 \mathrm{H}$ ), $4.68(\mathrm{~d}, J=17.4 \mathrm{~Hz}, 1 \mathrm{H}), 2.37(\mathrm{~s}, 3 \mathrm{H}), 2.14(\mathrm{~d}, J=3.9 \mathrm{~Hz}, 1 \mathrm{H}) .{ }^{13} \mathrm{C} \mathrm{NMR}\left(\mathrm{CDCl}_{3}\right.$, $125 \mathrm{MHz})$ 145.3, 139.6, 138.3, 135.7, 135.5, 132.6, 130.2, 127.2, 125.3, 125.1, 125.0, 124.6, 118.9, 115.5, 113.0, 66.8, 21.8. IR ( NaCl, $\left.\mathrm{cm}^{-1}\right): 3550,3089,3033,2980,2922$, 1596, 1411, 1369, 1292, 1246, 1188, 1174, 1138, 1094, 1026, 1016, 990, 921, 812, 795, 757, 705, 664. HRMS (TOF ES+) calculated for $\mathrm{C}_{22} \mathrm{H}_{21} \mathrm{O}_{3} \mathrm{NSNa}(\mathrm{M}+\mathrm{Na})^{+} 402.1140$, observed 402.1137.

\section{Cycloadduct (13)}

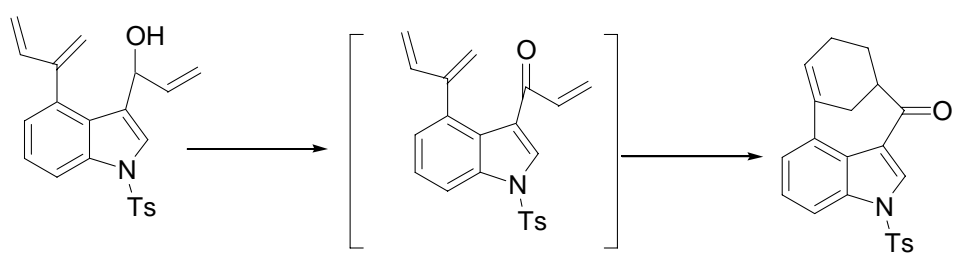

The alcohol (20 mg) was dissolved in $17 \mathrm{~mL}$ of $\mathrm{CH}_{2} \mathrm{Cl}_{2}$. The reaction mixture was magnetically stirred under $\mathrm{N}_{2}$ and about $100 \mathrm{mg}$ of activated $\mathrm{MnO}_{2}$ was added. After $1 \mathrm{~h}$, the reaction mixture was filtered through celite 545, and concentrated in vacuo to give the enone as a colorless tar. The enone (it is possible to isolate the enone in $64 \%$ at this stage) was dissolved in $45 \mathrm{~mL}$ of toluene and the solution was placed in a $450 \mathrm{~mL}$ Parr high pressure reactor. The solution was sparged with $\mathrm{N}_{2}$ for $10 \mathrm{~min}$, and the reactor was sealed. The reaction was heated to $120^{\circ} \mathrm{C}$ for $1 \mathrm{~h}$, and then the solvents were removed in vacuo. The colorless residue was purified by $\mathrm{SiO}_{2}$ flash chromatography using 3:1 hexanes:EtOAc to give the product as a white solid. Yield: $10 \mathrm{mg}, 50 \%$. Mp: $112.3-119.3^{\circ} \mathrm{C}$. ${ }^{1} \mathrm{H}$ NMR (CDCl, $\left.500 \mathrm{MHz}\right) 8.24$ (s, $\left.1 \mathrm{H}\right), 7.87$ (app.d, $\left.J=8.6 \mathrm{~Hz}, 2 \mathrm{H}\right), 7.81$ (dd, $J=$ 8.6, 0.73 Hz, $1 \mathrm{H}), 7.27-7.34$ (m, $3 \mathrm{H}), 7.16$ (dd, $J=7.2,0.61 \mathrm{~Hz}, 1 \mathrm{H}), 6.09-6.13$ (m, 1 H), 3.25-3.30 (m, $1 \mathrm{H}$ ), 2.80 (app.d, $J=13.2 \mathrm{~Hz}, 1 \mathrm{H}$ ), 2.46-2.54 (m, $1 \mathrm{H}), 2.39$ (s, $3 \mathrm{H}$ ), 2.29-2.36 (m, $2 \mathrm{H}), 1.97-2.06$ (m, $1 \mathrm{H}), 1.47-1.55$ (m, $1 \mathrm{H}) .{ }^{13} \mathrm{C} \mathrm{NMR}\left(\mathrm{CDCl}_{3}, 125 \mathrm{MHz}\right)$ 
202.8, 146.0, 139.8, 135.0, 133.9, 132.5, 131.0, 130.4, 127.51, 127.47, 126.2, 125.7, 123.5, 117.8, 111.7, 47.3, 31.5, 29.2, 23.3, 21.9. IR $\left(\mathrm{KBr}, \mathrm{cm}^{-1}\right)$ : 3119, 3091, 3031, 2947, 2894, 2870, 1658, 1596, 1513, 1481, 1422, 1377, 1189, 1178, 1132, 1096, 1025, 995, 856, 761, 704, 668, 579, 550. HRMS (TOF ES+) calculated for $\mathrm{C}_{22} \mathrm{H}_{19} \mathrm{O}_{3} \mathrm{NSNa}$ $(\mathrm{M}+\mathrm{Na})^{+}$400.0983, observed 400.0986.

\section{4-Furan-3-yl-1H-indole-3-carbaldehyde (14)}

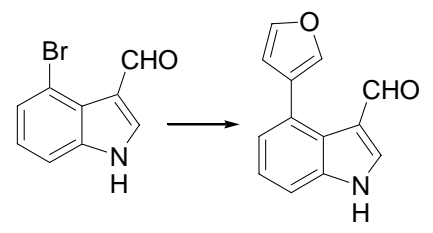

A $15 \mathrm{~mL}$ flask was fitted with a condenser, Ar inlet, and magnetic stir bar. This set-up was charged with 4-bromo- $1 \mathrm{H}$-indole-3-carbaldehyde (300 mg, $1.36 \mathrm{mmol}), \mathrm{Pd}\left(\mathrm{PPh}_{3}\right)_{4}$ (62.5 mg, $0.05 \mathrm{mmol}$ ), furan-3-boronic acid (200 mg, $1.8 \mathrm{mmol}$ ), DME (5 mL), and $\mathrm{Na}_{2} \mathrm{CO}_{3}(1.5 \mathrm{~mL}$ of a saturated solution in water). This heterogeneous mixture was heated to reflux for $3.5 \mathrm{~h}$, cooled, and then partitioned between EtOAc and water. The organic layer was washed three times with brine, dried over $\mathrm{Na}_{2} \mathrm{SO}_{4}$, filtered, adsorbed onto about 3 g of celite 545, and concentrated in vacuo. The material was purified via $\mathrm{SiO}_{2}$ flash chromatography using 2:1 hexanes:EtOAc as the eluent to give the product as a semisolid.

Yield: $0.30 \mathrm{~g}(100 \%)$.

${ }^{1} \mathrm{H} \mathrm{NMR}_{\left(\mathrm{DCCl}_{3}\right): \delta} 9.97$ (s, $\left.1 \mathrm{H}\right), 9.78$ (br.s, $\left.1 \mathrm{H}\right), 8.04$ (d, $\left.J=3.2 \mathrm{~Hz}, 1 \mathrm{H}\right), 7.62$ (dd, $J=$ 1.6, $0.8 \mathrm{~Hz}, 1 \mathrm{H}$ ), 7.54 (t, $J=1.7 \mathrm{~Hz}, 1 \mathrm{H}$ ), 7.45 (dd, $J=8.1,1.0 \mathrm{~Hz}, 1 \mathrm{H}$ ), 7.30 (app.t, $J=$ $7.6 \mathrm{~Hz}, 1 \mathrm{H}), 7.21$ (dd, $J=7.1,1.0 \mathrm{~Hz}, 1 \mathrm{H}), 6.64$ (dd, $J=1.7,0.7 \mathrm{~Hz}, 1 \mathrm{H}$ ). ${ }^{13} \mathrm{C} \mathrm{NMR}$ $\left(\mathrm{DCCl}_{3}\right): \delta 187.3,143.3,140.2,137.3,131.6,126.20,126.16,124.8,124.6,123.6,119.6$, 112.1, 111.8. IR (NaCl, $\left.\mathrm{cm}^{-1}\right)$ : 3243, 3056, 3000, 2963, 2940, 2091, 2863, 2850, 2767 , 1651, 1514, 1494, 1422, 1387, 1356, 1332, 1290, 1266, 1234, 1184, 1158, 1138, 1120 , 1062, 1046, 1022, 988, 873, 832, 788, 749, 663, 646, 599. HRMS (TOF ES+) calculated for $\mathrm{C}_{14} \mathrm{H}_{9} \mathrm{O}_{2} \mathrm{~N}(\mathrm{M}+\mathrm{H})^{+}$212.0712, observed 212.0705.

\section{4-Furan-3-yl-1-(toluene-4-sulfonyl)-1H-indole-3-carbaldehyde (15)}

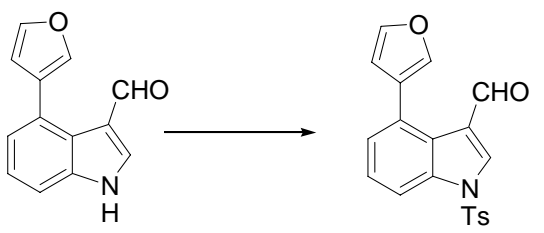


A 2-dram vial was charged with a solution of 4-furan-3-yl- $1 H$-indole-3-carbaldehyde (274 mg, $1.3 \mathrm{mmol}$ ) in $10 \mathrm{~mL}$ of $\mathrm{CH}_{2} \mathrm{Cl}_{2}$. The solution was magnetically stirred under $\mathrm{N}_{2}$ as DMAP (about $20 \mathrm{mg}, 0.16 \mathrm{mmol}$ ), triethylamine (265 $\mu \mathrm{L}, 1.9 \mathrm{mmol}$ ), and pTSCl (292 $\mathrm{mg}, 1.5 \mathrm{mmol}$ ) were added. The resulting yellow solution was stirred for $1 \mathrm{~h} 40 \mathrm{~min}$, and then was quenched with $1 \mathrm{~N} \mathrm{HCl}$ and diluted with more $\mathrm{CH}_{2} \mathrm{Cl}_{2}$. The organic layer was dried over $\mathrm{Na}_{2} \mathrm{SO}_{4}$, adsorbed onto celite 545 , and purified via $\mathrm{SiO}_{2}$ flash chromatography using 4:1 to 3:1 hexanes:EtOAc to give the product as an orange-tinted semi-solid. $\mathrm{Rf}=$ 0.23 in 3:1 hexanes:EtOAc (UV active and stained grey-green with anisaldehyde).

Yield: $400 \mathrm{mg}(84 \%)$.

${ }^{1} \mathrm{H} \mathrm{NMR}\left(\mathrm{DCCl}_{3}\right): \delta 9.85$ (s, $\left.1 \mathrm{H}\right), 8.35$ (s, $\left.1 \mathrm{H}\right), 8.01$ (dd, $\left.J=8.5,0.8 \mathrm{~Hz}, 1 \mathrm{H}\right), 7.88$ (app.d, $J=8.6 \mathrm{~Hz}, 2 \mathrm{H}$ ), 7.55 (dd, $J=1.5,1.0 \mathrm{~Hz}, 1 \mathrm{H}$ ), 7.53 (t, $J=1.7 \mathrm{~Hz}, 1 \mathrm{H}$ ), 7.39 (dd, $J=8.5,7.5 \mathrm{~Hz}, 1 \mathrm{H}$ ), 7.31 (app.d, $J=8.5 \mathrm{~Hz}, 2 \mathrm{H}$ ), 7.25 (dd, $J=7.3,1.0 \mathrm{~Hz}, 1 \mathrm{H}$ ), 6.53 (dd, $J=1.7,1.0 \mathrm{~Hz}, 1 \mathrm{H}), 2.39$ (s, $3 \mathrm{H}) .{ }^{13} \mathrm{C} \mathrm{NMR}\left(\mathrm{DCCl}_{3}\right): \delta$ 186.8, 146.4, 143.7, 140.2, 135.6, 134.6, 131.6, 130.5, 127.6, 127.0, 126.56, 126.49, 125.50, 125.35, 122.6, 113.2, 111.9, 21.9. IR ( $\left.\mathrm{NaCl}, \mathrm{cm}^{-1}\right)$ : 3133, 3055, 2923, 2870, 1677, 1596, 1527, 1506, 1406, 1378, 1276, 1192, 1175, 1160, 1125, 1096, 1087, 1030, 1006, 930, 874, 834, 813, 801, 788, 752, 737, 703, 679, 661, 615, 579. HRMS (TOF ES+) calculated for $\mathrm{C}_{20} \mathrm{H}_{15} \mathrm{O}_{4} \mathrm{NSNa}(\mathrm{M}+\mathrm{Na})^{+}$388.0620, observed 388.0603.

\section{1-[4-Furan-3-yl-1-(toluene-4-sulfonyl)-1H-indol-3-yl]-prop-2-en-1-ol (16)}

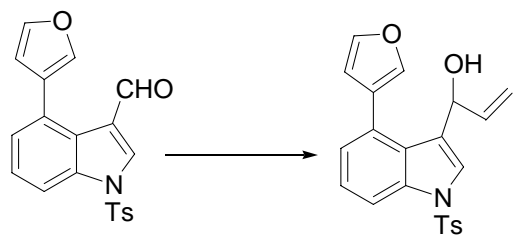

4-Furan-3-yl-1-(toluene-4-sulfonyl)-1H-indole-3-carbaldehyde (175 mg, $0.48 \mathrm{mmol}$ ) was dissolved in $1.0 \mathrm{~mL}$ of dry THF under $\mathrm{N}_{2}$ in a 2-dram vial. The solution was magnetically stirred and cooled in a dry ice-acetone bath as vinylmagnesium bromide ( $0.60 \mathrm{~mL}$ of a $1.0 \mathrm{M}$ solution in THF) was added dropwise. The mixture was stirred in the cool bath for $15 \mathrm{~min}$, and then the dry ice bath was replaced with an ice bath and stirring was continued for 15 additional minutes. The reaction mixture was quenched with saturated aqueous $\mathrm{NH}_{4} \mathrm{Cl}$ and extracted into EtOAc. The organic layer was washed with brine, dried over $\mathrm{Na}_{2} \mathrm{SO}_{4}$, and concentrated in vacuo. The residue was purified via $\mathrm{SiO}_{2}$ flash chromatography using 3:1 to 2:1 hexanes:EtOAc to give the product as an oil. The product eluted between the starting material and the reduction product.

Yield: $190 \mathrm{mg}$ (100\%).

${ }^{1} \mathrm{H} \mathrm{NMR}\left(\mathrm{DCCl}_{3}\right): \delta 8.06$ (d, $\left.J=8.3 \mathrm{~Hz}, 1 \mathrm{H}\right), 7.86(\mathrm{~d}, J=8.3 \mathrm{~Hz}, 2 \mathrm{H}), 7.68(\mathrm{~s}, 1 \mathrm{H})$, 7.58 (app. s, 1 H), 7.57 (t, $J=1.7 \mathrm{~Hz}, 1 \mathrm{H}$ ), 7.37 (t, $J=7.9 \mathrm{~Hz}, 1 \mathrm{H}$ ), 7.31 (app. d, J = 9.5 Hz, 2 H), 7.17 (d, $J=7.3$ Hz, 1 H), 6.63-6.64 (m, 1 H), 6.05 (ddd, $J=17.1$, 10.5, 5.4 Hz, 
$1 \mathrm{H}$ ), 5.35 (app. t, $J=4.5 \mathrm{~Hz}, 1 \mathrm{H}), 5.21-5.27$ (m, $2 \mathrm{H}) . \quad \mathrm{IR}\left(\mathrm{NaCl}, \mathrm{cm}^{-1}\right)$ : 3550, 3418, 3146, 3090, 3070, 3035, 2983, 2924, 1644, 1596, 1557, 1505, 1494, 1479, 1446, 1414, 1372, 1308, 1291, 1245, 1189, 1172, 1127, 1095, 1031, 1006, 937, 908, 874, 813, 788, 757, 737, 704, 666, 617, 600, 579. HRMS (TOF ES+) calculated for $\mathrm{C}_{22} \mathrm{H}_{19} \mathrm{O}_{4} \mathrm{NSNa}$ $(\mathrm{M}+\mathrm{Na})^{+}$416.0933, observed 416.0928.

\section{Cylcoadduct (18)}

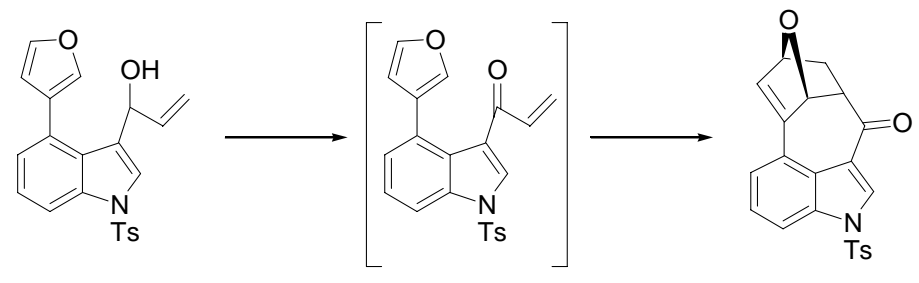

The alcohol (19.7 mg) was dissolved in $15 \mathrm{~mL}$ of $\mathrm{CH}_{2} \mathrm{Cl}_{2}$. The reaction mixture was magnetically stirred under $\mathrm{N}_{2}$ and $80 \mathrm{mg}$ of activated $\mathrm{MnO}_{2}$ was added. After $40 \mathrm{~min}$, the reaction mixture was filtered through celite 545 , and concentrated in vacuo. The residue was dissolved in $45 \mathrm{~mL}$ of toluene, and the solution was placed in a $450 \mathrm{~mL}$ Parr high pressure reactor. The solution was sparged with $\mathrm{N}_{2}$ for $10 \mathrm{~min}$. The reactor was sealed and heated at $120^{\circ} \mathrm{C}$ for $3 \mathrm{~h}$. The solvent was then removed in vacuo at less than $35^{\circ} \mathrm{C}$, and the brown residue was purified by $\mathrm{SiO}_{2}$ flash chromatography using 3:1

hexanes:EtOAc. The product $(\mathrm{Rf}=0.1)$ was collected as a tan solid. Yield: $13.5 \mathrm{mg}$ (69\%).

Mp: $185-190{ }^{\circ} \mathrm{C}$, decomp.

${ }^{1} \mathrm{H} \mathrm{NMR}\left(\mathrm{CDCl}_{3}, 500 \mathrm{MHz}\right) 8.27$ (s, $\left.1 \mathrm{H}\right), 7.85-7.91$ (m, $\left.3 \mathrm{H}\right), 7.37$ (t, $\left.J=7.8 \mathrm{~Hz}, 1 \mathrm{H}\right)$, 7.31 (d, $J=8.6 \mathrm{~Hz}, 2 \mathrm{H}), 7.23$ (d, $J=7.3 \mathrm{~Hz}, 1 \mathrm{H}$ ), 6.31 (d, $J=1.5 \mathrm{~Hz}, 1 \mathrm{H}), 5.14-5.18$ (m, $2 \mathrm{H}$ ), 3.42 (dt, $J=10.1,4.3 \mathrm{~Hz}, 1 \mathrm{H}$ ), 2.71 (ddd, $J=11.5,10.1$, $4.4 \mathrm{~Hz}, 1 \mathrm{H}$ ), 2.39 (s, $3 \mathrm{H}), 1.51$ (dd, $J=11.5,4.4 \mathrm{~Hz}, 1 \mathrm{H}) .{ }^{13} \mathrm{C} \mathrm{NMR}\left(\mathrm{CDCl}_{3}, 125 \mathrm{MHz}\right) 196.6,146.3,143.7$, 134.7, 133.9, 133.2, 132.2, 130.5, 130.0, 127.6, 126.0, 124.2, 122.8, 120.6, 112.7, 80.02, 79.97, 50.1, 35.4, 21.9. IR (KBr, $\left.\mathrm{cm}^{-1}\right): 3145,3085,3064,3047,3031,2995,2981,2951$, 1644, 1596, 1517, 1484, 1426, 1376, 1287, 1191, 1182, 1160, 1096, 922, 818, 813, 788, 757, 704, 694, 665, 612, 576, 549. 